\title{
Sufrir de azúcar por ak'chamel 'mal echado' en el marco de un horizonte cosmológico amplio: dos casos en Tenejapa, Chiapas
}

\section{Suffering from «Sugar» due to Ak'chamel (An Evil Spell) within the Framework of a Wide Cosmological Horizon: Two Cases in Tenejapa, Chiapas}

Jaime Tomás Page Pliego Centro de Investigaciones Multidisciplinarias sobre Chiapas y la Frontera Sur-UNAM email: jaimepagepliego@gmail.com ORCID: orcid.org/: 0000-0003-0508-0647

Resumen:

A partir de entrevistas realizadas a diabéticos de tres localidades de los Altos de Chiapas y del seguimiento de casos, en este trabajo se hace referencia a la subjetividad expresada por dos mujeres que consideran que su padecimiento de «azúcar» fue causado por ak'chamel 'mal echado'. Los casos fueron seleccionados de entre 16 enfermos tseltales de la cabecera municipal de Tenejapa, Chiapas. Se trata de mujeres mayores de 45 años, monolingües y analfabetas; la asociación causal se cimenta en ciertos rasgos de sus sueños, aunados a condiciones particulares de vida caracterizada por violencia constante, o bien signos y síntomas particulares.

Palabras clave: diabetes, mal echado, subjetividad.

Abstract:

Based on interviews made with people suffering diabetes from three different locations in the Chiapas Highlands (los Altos de Chiapas) and follow-up cases, this paper makes reference to the subjectivity expressed by two women who believed that their «sugar» ailment had been caused by ak'chamel (an evil spell). The cases were selected among 16 Tseltal women with diabetes from the municipal head of Tenejapa, Chiapas State. Aged over 45, they are monolingual and illiterate; the causal association is based on certain features appearing in their dreams together with specific life conditions characterized by ongoing violence, or specific signs and symptoms.

Keywords: diabetes, evil spell, subjectivity. 


\section{Introducción}

os datos de este artículo provienen de un proyecto de investigación enfocado en representaciones sociales y estrategias de atención en torno a la diabetes mellitus en la población que habita la periferia de la ciudad de San Cristóbal de Las Casas — mayahablantes y ladinos'—, y cabeceras municipales de San Juan Chamula —-tsotsiles- y Tenejapa -tseltales y ladinos-. Las tres son localidades de la región Altos de Chiapas.

En dicho estudio, efectuado entre 2008 y 2015, se trabajó con personas que viven con diabetes y que por lo general no acuden a los servicios públicos de salud, o que lo hacen en forma eventual. Esto se explica por la experiencia de largo plazo que los grupos originarios han sufrido al demandar atención en dicho sistema y que ha dado lugar a vastas narrativas y testimonios por parte de los usuarios tanto originarios como mestizos, la mayoría de desencanto, respecto de los servicios de salud, entre los que destaca por su frecuencia: racismo manifiesto en violencia verbal, «regaños», grandes dificultades para la comunicación debido a que el personal de salud no habla ni entiende la lengua, elevada mortandad al recurrir a las instituciones biomédicas, esterilizaciones sin consentimiento de las mujeres y sus familiares, falta de medicamentos, que se niegue la atención subsecuente a enfermos por no ajustarse a las indicaciones recibidas, que los médicos asignados tengan escasa formación y experiencia y que por ello sea común que no ayuden a las personas a sanar o las dañen con sus tratamientos. Y así se podría continuar la lista (véase Page 2002:96, 141-143).

El objetivo general fue documentar y analizar, por medio de entrevistas semiestructuradas y seguimiento de trayectorias, cómo se construyen las representaciones y prácticas en torno a la diabetes mellitus no insulinodependiente (DMNID), cómo se actualizan (deconstruyen/reconstruyen) a partir de la díada: experiencia de los enfermos-influencia del entorno social —que se suscita durante el proceso de búsqueda de atención - y qué prognosis se presentan —considerando en dicho proceso al enfermo, su familia nuclear y las redes sociales en que están insertos-. Ello, en el marco de las relaciones de hegemonía/subalternidad y dominación que se imponen a los participantes.

1 Distinguimos entre mestizo y ladino de la siguiente manera: mestizos son aquellas personas que no viven en contacto cotidiano con población originaria; ladinos son aquellos mestizos que viven en regiones donde predomina la población originaria, con la que interaccionan cotidianamente. 
Entre los hallazgos detectamos un sector constituido por mujeres mayores de 40 años, monolingües, analfabetas y tradicionalistas, que atribuyeron como causa de la diabetes haber sido objeto de ak'chamel, ya fuera por envidias o disputas. Esto en un contexto en el que la enfermedad se percibe inscrita en un horizonte (Jodelet 2008) amplio, que da lugar al uso de recursos varios y a una atribución cambiante de las causas, aspecto del que se hablará más adelante con mayor detenimiento. De ese modo se busca mostrar las particularidades en cuanto a la atribución de las causas del "azúcar» y las características que adquieren las trayectorias en estos casos, mediante la presentación de dos en particular.

Con objeto de contextualizar, se muestran algunos antecedentes, la metodología empleada en la investigación, conceptos principales y aspectos teóricos que orientaron la pesquisa y algunos antecedentes.

\section{Antecedentes}

La diabetes mellitus (DM) se presenta cuando la hormona denominada insulina acusa por agotamiento o deterioro una deficiente o nula producción en las células beta del páncreas como resultado de concentraciones crecientes de glucosa en sangre, debido a un problema de resistencia a la hormona a nivel de los receptores específicos presentes en las superficies celulares de músculo y panículo adiposo, lo que a su vez genera la hiperproducción de insulina que, a la larga, lleva al deterioro de las células beta y a la disminución o ausencia de su producción. La hiperglucemia, consecuencia de ello, acarrea trastornos metabólicos característicos de la diabetes mellitus (German 2013:13).

En los avances de la séptima edición del Atlas de la International Diabetes Federation (IDF 2015) se señalaba que 415 millones de personas tenían diabetes, 35 millones más de lo que en 2006 se calculaba para 2036, y se presume que para 2040 habrá 642 millones de diabéticos; dicha predicción se completa al informar que una de cada 10 personas en el mundo vivirá con DM (IDF 2015: mensaje clave). De los 415 millones contabilizados en 2015, es decir, uno de cada 11 diabéticos, $46.5 \%$, casi la mitad, estaban sin diagnóstico. Otro dato de nuestro interés señala que $75 \%$ de los diabéticos habita en países de ingresos medios y bajos (IDF 2008).

Además, en las tres ediciones de dicho Atlas IDF (2008, 2013 y 2015) se destaca que la DMNID es más frecuente en los países pobres, sobre todo porque en ese 
ámbito se recurre en exceso a comidas hipercalóricas de bajo costo y bebidas gaseosas azucaradas.

La diabetes mellitus constituye uno de los más importantes problemas de salud pública a nivel nacional. En Chiapas se situaba, entre 2008 y 2012, como una de las primeras causas de mortalidad. Al respecto cabe destacar que, en el estado, en el periodo previo a 2003 fue notorio cómo la DM no apareció entre las causas más frecuentes de casos nuevos. Es a partir del año 2004 que esta enfermedad despunta de manera elevada, para irse incrementando hasta 2007. En 2008 mostró una disminución. En lo que toca a Tenejapa y los municipios de Chamula y San Cristóbal de Las Casas, al recabar datos en las actas de defunción de las tres localidades constatamos que durante el periodo señalado, la diabetes mellitus fue la primera causa de muerte en los tres municipios.

Según reporte del INEGI, de las 18287 defunciones registradas en el estado de Chiapas en 2006, la DM se ubicó como la tercera causa fatal, después de tumores malignos y enfermedades del corazón, con 1774 defunciones, 9.7\% del total de mortalidad y una tasa de 46.43 por 100000 (INEGI 2006).

En 2008 prevaleció el mismo orden en cuanto a defunciones: de 19570 decesos, 2005 corresponden a DM, es decir, $10.24 \%$, porcentaje superior al registrado en 2006, con tasa por 100000 de 44.07, levemente inferior a la precedente (ISCH 2008).

Por otro lado, al analizar las causas de mortalidad por sexo para dicho año podemos notar que de los decesos de mujeres, 13\% del total ocurrió por DM, con lo cual se situaba como segunda causa de muerte después de tumores malignos, que ascendió a 15\%. Con ello se confirmaba, según se asienta en diferentes textos sobre la materia, primero, que mueren más mujeres que hombres por diabetes mellitus $y$, segundo, que por sexo y para Chiapas, este padecimiento fue la segunda causa de muerte en mujeres en ese año (ISCH 2008).

\section{Transfiguraciones}

Respecto de las etnomedicina (Page 2010, 2011), los pueblos originarios mesoamericanos han logrado sobrevivir al embate de la invasión española por más de 500 años, no sin menoscabos, en parte gracias al conocimiento que han pragmáticamente adquirido para resolver los problemas de salud y enfermedad, lo cual ha supuesto la incorporación de elementos de las medicinas de los grupos domi- 
nantes, procesados y resignificados desde su propia racionalidad, y trasmitidos como complejo de conocimientos y experiencias de generación en generación y, por otra parte, haciendo uso de los escasos servicios de atención médica que el Estado les ha provisto en diferentes momentos y con diversos grados de calidad, así como de la biomedicina privada y de las distintas medicinas populares.

Cabe señalar que desde la colonia hasta nuestros días los sistemas etnomédicos han perseverado debido a la enorme utilidad que reportan a los grupos que los detentan. Los recursos de que han hecho acopio a lo largo del tiempo, aunados a su capacidad de actualización y adaptación, han abonado en beneficio de los conjuntos sociales en que tienen lugar sus prácticas, lo que les ha permitido en el pasado y en el presente sobrepasar las persecuciones policiacas y jurídicas, así como las emprendidas desde los ámbitos biomédico estatizado y del cristianismo católico y protestante, variando la intensidad de la violencia ejercida según los vaivenes de lo político y los intereses de los conjuntos sociales dominantes.

Entre los mayas de los Altos de Chiapas se ha documentado (Page 2010, 2011) que las etnomedicinas no se han constituido como entidades inamovibles, ciertamente fundamentadas en visiones del mundo que tampoco han sido estáticas, como se observa en publicaciones en las décadas de 1960 y 1980 (Díaz 1963, Holland 1963, Guiteras 1965, Hermitte, 1970, Fábrega 1973, Gossen 1975, 1980, Pozas 1977, Köhler 1995), sino que están forzadas a cambios constantes condicionados por su posición subordinada en el ámbito de los procesos de hegemonía-subalternidad, que las obliga a una reformulación constante en aras de minimizar la violencia de que son objeto para no verse condenadas a desparecer.

En este sentido, el impacto neocolonial inserto en la escalada de la globalidad neoliberal, y específicamente del proselitismo protestante descalificador y satanizador, ha generado en el corto plazo transfiguraciones ${ }^{2}$ en las formas de concebir y vivir el mundo en la población que nos ocupa. De entre las transfiguraciones y los cambios que han tenido lugar destacan, en primer término, la reciente transformación ideológico-religiosa ocurrida entre buena parte de tsotsiles y tseltales, principalmente como consecuencia del embate proselitista de grupos cristianos tanto protestantes como católicos, que inició en la década de los setenta y se

2 Entendiendo «transfiguración étnica» como los procesos mediante los cuales, como señalan Bartolomé y Barabas, se realizan «[...] sucesivas alteraciones internas tanto biológicas (mestizajes) como culturales, y en sus formas de relación con la sociedad envolvente» entre los grupos étnicos, en un intento por mantenerse como entidades étnicas ante el embate de los diferentes agentes de la sociedad dominante. «Es decir, que la transfiguración supone la existencia de cambios adaptativos que cambian la fisonomía cultural» (Bartolomé y Barabas 1996:28.) 
viralizó en los ochenta; dicha acción se centró de manera importante en la satanización y descalificación de las prácticas religiosas y etnomédicas de mayas y zoques, cuyo resultado, desde de la perspectiva hegemonía/subalternidad y dominación, ha sido una necesaria transformación estructural y una reformulación de los aspectos perseguidos a efecto de prevalecer antes que desaparecer (Page 2011:114).

Por ejemplo, un cambio significativo derivado del impacto de la racionalidad biomédica que permea en todos los conjuntos sociales ha consistido en que en las dos últimas décadas quienes detentan el cargo de ak'vomol 'hierbatero' han ganado preeminencia sobre los j-iloletik-ch'abajeletik 'quienes sienten y escuchan las palabras que circulan en la sangre', puesto que tiende a privilegiarse el uso de los recursos que los primeros manejan por encima de los rezos, es decir, va permeando una concepción que sitúa la enfermedad predominantemente en el ámbito natural. Puede mencionarse, entre esos factores, la presencia constante en las comunidades de agentes de la sociedad dominante que antaño estuvieron ausentes o fueron rechazados y que en la actualidad han ido influyendo sobre los jóvenes mayas, cuya mayoría se ha desarrollado en contextos diferentes a los de sus padres y abuelos. Entre estos factores pueden mencionarse: diferentes niveles escolares que van desde pre-escolar hasta bachillerato; farmacias y consultorios médicos y odontológicos atendidos por j-loktores (ancianos que fungieron como enfermeros en lo que fuera el Instituto Nacional Indigenista), promotores y técnicos dentales, biomédicos titulados; televisión, diferentes opciones de estaciones de radio en español, en tsotsil y en tseltal, entre las cuales pululan las de corte religioso; la influencia en las formas de concebir y vivir el mundo como resultado de la migración en busca de trabajo en la región, en otros estados del país o el extranjero, por citar algunos.

A través de estos agentes institucionales o empresariales se transmiten las posturas hegemónicas sobre el deber ser de las personas, lo que en el corto plazo ha modificado sensiblemente la visión del mundo en amplios sectores de las poblaciones que nos ocupan. La racionalidad biomédica ha ido adquiriendo entonces una importancia que en el pasado no tenía; además, las diferentes opciones de atención son utilizadas con mayor libertad e incluso combinadas.

Sin embargo, es claro que a partir de la comparación de los procesos a que se ha visto sometida la población y con ello sus etnomedicinas, se puede observar la gradual extinción de ch'abajeletik tseltales y j-iloletik tsotsiles: 
van desapareciendo las formas de atención que durante las últimas décadas han mantenido las medicinas de los mayas de los Altos, primero en aquellas localidades donde los jóvenes ya no sueñan y que sobrevendrá con la muerte de los ancianos que actualmente se ocupan de la atención, y más adelante en aquellas en que cada vez son menos los jóvenes que reciben el mandato, lo cual es sinónimo de un cambio drástico (Page 2011:343).

Lo dicho apunta al predominio de herbolarios, hueseros y parteras como resabios de las etnomedicinas. De hecho, esto es un común denominador en el municipio de Oxchuc y de manera importante en Tenejapa, donde es muy difícil encontrar un ch'abajel, y a quienes se busca es a conocedores de la herbolaria o de la biomedicina. Además, por ejemplo, en Oxchuc se ha observado que lo relativo a ak'chamel es actualmente atendido por «espiritistas» (Page 2010:121).

\section{Metodología}

La selección de colaboradores, personas que viven con diabetes, familiares inmediatos y curadores de distintos sistemas médicos, se realizó mediante el procedimiento denominado bola de nieve, ya fuera estableciendo los contactos a través de conocidos o bien captando a aquellos que voluntariamente se acercaran a los investigadores. A los sujetos que accedieron a participar en el proyecto, previo consentimiento informado, se les entrevistó sobre la base de una guía semiestructurada, orientada a los aspectos fundamentales de interés para la investigación.

En Tenejapa se contactó a 16 personas que viven con diabetes, 14 en Chamula y 22 en San Cristóbal de Las Casas. De entre estos a 14 se les acompañó en las trayectorias para el control del padecimiento durante dos años o hasta su deceso.

Las entrevistas en español, capturadas en audio, fueron realizadas por los investigadores, en tanto que para las entrevistas en lenguas tsotsil y tseltal se contó con apoyo de intérpretes, con quienes previamente se trabajó con objeto de sensibilizarlos y lograr el manejo fluido y pertinente del instrumento; luego hicieron la traducción en audio y algunas transcripciones. Todas las entrevistas realizadas en español y algunas de las grabadas fueron recopiladas por el investigador.

La entrevista a enfermos y a sus familiares se centró en abordar las circunstancias que dieron origen a la DM, cómo se explicaban en ese momento y en diferentes etapas su padecimiento; narración de la trayectoria, experiencias en 
torno a tratamientos, dieta y actividad física; aspectos de orden socioeconómico, cultural, religioso y el impacto de todo ello en cada caso.

Si bien se pretendía dar seguimiento a las trayectorias con colaboradores de las tres localidades, esto solo fue posible principalmente con ocho en Tenejapa, cinco en San Cristóbal y uno en Chamula; en este último caso debido a dos situaciones: porque los participantes se negaron a ello y porque esperaban apoyos para el control de la diabetes que no estaba en nuestras manos proporcionarles.

El registro del seguimiento se realizó mediante notas de campo, y en algunos casos con fotografías.

Por último, cabe advertir que, a petición de los colaboradores, los nombres que se utilizan son todos ficticios.

\section{Marco conceptual y teórico}

Los conceptos centrales para el abordaje del tema son el de «representaciones sociales» y lo que Menéndez (2002:135) ha denominado «teoría de las prácticas». El problema en estudio se acometió principalmente desde «el punto de vista de los actores significativos»: los enfermos que no recurren o no lo hacen regularmente a los servicios de salud. El otro concepto o noción es la de «horizonte amplio».

A partir de lo documentado en torno a las prácticas para la conservación de la salud, así como en lo tocante a la búsqueda de atención para el control de las enfermedades en general y de las crónicas en particular, y considerando que los pueblos originarios con resabios mesoamericanos acceden de manera exclusiva, alternada o al mismo tiempo, a las distintas opciones de atención de que disponen a nivel local y regional, sin que ello les implique conflicto de orden ético, moral o conceptual a nivel personal familiar o social, para la comprensión de lo observado y su análisis se propone un modelo que se constituye a partir de la subjetividad/intersubjetividad inmersos en un contexto particular transubjetivo (Jodelet 2008) que da lugar a un «pluralismo médico» (Menéndez 2003:16), que implica un «horizonte amplio».

En tal sentido, con Jodelet (2008) entiendo que la esfera de la subjetividad remite a la apropiación/encarnación y elaboración de la experiencia del individuo fundada en procesos cognitivos, emocionales y físicos, entre lo que se enfatiza, además de la palabra, la acción no verbalizada como medio de transmisión de 
conocimientos (Ortner 2006, citado en Good-Eshelman 2015:145), por medio de los cuales se configuran/asimilan, transforman o cambian las representaciones de todo orden (Jodelet 2008:51-52).

Cabe además establecer que el pensamiento $y$, por tanto, la subjetividad a que da lugar, aunque sin ser reductible al hecho, tiene un «encadenamiento histórico», es decir, que lo social histórico "constituye la condición esencial de la existencia del pensamiento y la reflexión» (Castoriadis 1997:3), cuyo contenido intrínseco y estructurado reside en la esfera transubjetiva.

A través de la esfera de la subjetividad nos acercamos a las formas en que los sujetos conciben y viven la salud, piensan y sufren (padecen) la enfermedad y perciben las ventajas o desventajas de los servicios y recursos de los diferentes sistemas médicos a que tienen acceso. Respecto de la atención: ¿cómo se posicionan en relación con los procedimientos utilizados por los distintos agentes de los sistemas médicos para el control o la curación de las enfermedades? En torno a las condiciones socioeconómicas y culturales: ¿cómo se ven afectados y cómo entienden los problemas de estos ámbitos, incluso lo religioso en el sentido de facilitar o dificultar el acceso a la atención y las terapéuticas?, y, por lo tanto, poniendo atención a los procesos mentales y emocionales en los que se ha introyectado y procesado la experiencia (Biehl et al. 2007:5) y que implican expectativas y estrategias a futuro.

La intersubjetividad, como puntualiza Jodelet, se refiere a «las diferentes conceptualizaciones sobre la relación con el otro y su interiorización por el sujeto», y cabe indicar que «el lugar del encuentro intersubjetivo se halla, como lo muestra Merleau-Ponty (2003), en el campo de la institución que significa» (Jodelet 2008:48).

Tanto el sujeto como el otro son inseparablemente instituido e instituyente. Yo me proyecto en él y él en mí, hay una proyección-introyección, productividad de lo que yo hago en él y de lo que él hace en mí, comunicación verdadera por arrastre lateral: se trata de un campo intersubjetivo o simbólico, el de los objetos culturales, que constituye nuestro medio ambiente, nuestra bisagra, nuestro punto de juntura (Merleau-Ponty 2003:35-38, citado en Jodelet 2008:49).

La intersubjetividad en nuestro caso hace referencia a los intercambios que se suscitan entre personas en torno al padecimiento, al padecer de azúcar, a «modos de influencia» resultado «de procesos de interacción y de negociación 
de sentido», que dan lugar a la «resignificación de la experiencia de los (sujetos) sociales» interactuantes (Jodelet 2008: 49, en paréntesis texto agregado por mí).

Estos modelos de intervención siempre hacen referencia a un trabajo sobre las representaciones individuales, sociales o colectivas. Tarea que supone la corrección de creencias consideradas como inadecuadas o falsas, la valorización de saberes de sentido común, la concientización crítica de las posturas ideológicas, la reinterpretación de situaciones de vida y la puesta en perspectiva de las posiciones en función de un análisis de los contextos de la acción y del punto de vista de los sujetos (Jodelet 2008:49-50).

Como también señala la autora, adentrándose en la esfera de la transubjetividad, «el lugar del encuentro intersubjetivo se sitúa, como lo muestra MerleauPonty (2003), en el campo de la institución que significa» —la biomédica o la etnomédica-, que remite a diversos órdenes, reglas de operación de la institución y agentes que llevan la voz (para nuestro caso: médicos, etnomédicos, religiosos, políticos), que interactúan con los usuarios del sistema fundamentalmente mediante esquemas verticalizados y coercitivos.

Y como señala Castoriadis:

Los individuos socializados son fragmentos hablantes y caminantes de una sociedad dada; y son fragmentos totales; es decir que encarnan -en parte efectivamente, en parte potencialmente- el núcleo esencial de las instituciones y de las significaciones de su sociedad. No hay oposición entre el individuo y la sociedad, el individuo es una creación social, a la vez en tanto tal y en su forma histórica cada vez (Castoriadis 1997:3-4).

Así, la esfera de la transubjetividad no solo configura la intersubjetividad, sino también la subjetividad, en un proceso de retroalimentación dialéctica, inmiscuyéndose en estos en todo momento, pero a la vez reestructurándose en dicha interacción. Al respecto, Jodelet dice:

En la formación de las representaciones sociales, la esfera de la transubjetividad se sitúa en relación con la intersubjetividad y con la subjetividad, y remite a todo lo que es común para los miembros de un mismo colectivo [...] Su escala abarca tanto a los individuos y a los grupos, como a los contextos de interacción, las producciones discursivas y los intercambios verbales (Jodelet 2008:53). 
De manera más dinámica, Castoriadis expresa respecto de la sociedad y sus instituciones:

La sociedad es creación, y creación de sí misma, autocreación. Es la emergencia de una nueva forma ontológica —un nuevo eidos - y un nuevo nivel de modo de ser. Es una cuasitotalidad cohesionada por las instituciones (lenguaje, normas, familia, modos de producción) y por las significaciones que estas instituciones encarnan (tótems, tabús, dioses, Dios, polis, mercancía, riqueza, patria, etcétera) ambas -instituciones y significaciones - representan creaciones ontológicas (Castoriadis 1997:4).

La transubjetividad, en lo que a nuestro tema toca, como ya se señaló, da cuenta de la dimensión-macrosocial-del-proceso/salud/enfermedad/atención-prevención (PSEA-P), ${ }^{3}$ en particular lo que atañe a las distintas instituciones públicas y privadas que operan y/o inciden en el PSEA-P con su correspondiente normativa, discursos, conceptos y prácticas que enmarcan el punto de vista hegemónico sobre la enfermedad en términos genéricos, y sobre las formas en que se administra la atención y la prevención, hechos que vislumbramos, cabe subrayar, desde una perspectiva inmersa en contextos de hegemonía/subalternidad/dominación. ${ }^{4}$

Los elementos de la esfera de la transubjetividad que circulan entre los conjuntos sociales que nos ocupan tienen lugar en lo que he denominado un «horizonte amplio», en referencia a un modelo que nos permite esquematizar cómo se concibe y se actúa en el proceso particular de salud/enfermedad/atención-prevención y la manera en que se ubican sus diferentes elementos. Un modelo que da cabida, a guisa de abanico, a la coexistencia de elementos diversos que van desde los religiosos con resabios prehispánicos, entremezclados con elementos cristianos; elementos de orden natural, cuyo rango gravita entre la noción de frío-calor hasta los de orden biomédico, inmersos todos en el continuo de una intersubjetividad en la que no se cuestionan racionalidades provenientes de diferentes paradigmas, porque el criterio fundamental de elección radica en el

3 La noción de proceso de salud/enfermedad/atención-prevención se refiere a que en los diferentes sistemas médicos, incluido el biomédico, lo ideológico y la visión del mundo determinan las formas que adoptan los procesos de salud-enfermedad-atención; es decir, las formas de estar sano, de enfermar, y la coherencia que se precisa entre ambas y las que adopta la atención para la solución de los diferentes problemas.

${ }^{4}$ Hegemonía concierne a la dirección política, cultural e ideológica que ejerce lo que se denomina la clase fundamental o dirigente sobre los conjuntos sociales subordinados a esta. Subordinados en función de que en la disputa por los términos de la relación hay acuerdos, consensos. La dominación, entonces, tiene lugar cuando la clase fundamental impone por la fuerza sus posturas (Portelli 1973). 
impacto positivo o negativo de que lo «utilizado» tenga en el control o la erradicación del problema de salud-enfermedad que se presenta, y clasifica en función de los elementos utilizados.

La clasificación de que hablamos, que solo ubica racionalmente, pero no se opone operativamente al uso de otros recursos, contiene, por un lado, un segmento referente a lo que se considera natural, en el que residen las nociones/ prácticas que buscan armonizar dentro de la propia racionalidad del grupo la dualidad frío-caliente inscrita en los diversos elementos tanto de la naturaleza como subjetivos, y los procesos de asimilación de elementos del cuidado de la salud inducidos desde la biomedicina (higiene personal, de la casa y la alimentación; prácticas en el cuidado de los niños, del embarazo, etc.), aunado a la racionalidad biomédica que gradualmente se impone.

A ello se suma un segmento espiritual en el que residen racionalidades y prácticas para la conservación de la salud y su recuperación, sobre todo de padecimientos graves, prácticas que implican rituales que exige cotidiana o periódicamente la religiosidad particular, considerando de entre estos los que deben llevarse a nivel individual, familiar y de petición a las fuerzas sobrenaturales, todo lo cual debe coadyuvar a la salud y la prosperidad. Mediante la realización de dichas ceremonias y rituales se cumple con lo exigido por el mandato primordial. ${ }^{5}$ Además, de respetar las costumbres y el orden establecido (Page 2011). En dicho segmento tenemos, por un lado, el ak'chamel (Page 2008, 2010, Sánchez 2013, Figuerola 2010), y por otro las consecuencias del incumplimiento del mandato primordial con el consecuente retiro de la protección que prodigan las deidades celestiales, lo que predispone a las personas al susto o a otras formas de pérdida del ch'ulel-alma, o bien crea condiciones para que esta entidad pueda ser atrapada por los diferentes entes sobrenaturales que pueblan la superficie de la tierra, así como otras vicisitudes que pueden acaecer a las personas por medio de sus otras entidades anímicas, lo que resulta en enfermedades prolongadas, graves e incluso la muerte. ${ }^{7}$

5 El mandato primordial remite a que, según establece el mito de origen, la humanidad fue creada por las deidades con el único propósito de que las nutran, las adoren y las sostengan (Page 2011).

6 Ak'chamel no es sinónimo de brujería. Posee características propias que lo distinguen de las prácticas mestizas y populares mexicanas, sobre todo por las formas de interceder valiéndose, el j-ak'chamel 'el que pone enfermedad' tenejapaneco de la entidad lab que es la capacidad de este para proyectar su entidad anímica constituida por diferentes humanoides, animales o fenómenos naturales; entidad de naturaleza gaseiforme, lo que le da capacidad de penetrar los cuerpos de sus víctimas para dañarlas (Figuerola 2010, Pitarch 1996).

7 Para mayor información al respecto consultar Page 2011, parte II, cap. 3, a partir de la p. 178. 
En el segmento natural se sitúan, por un lado, las nosologías de orden energético generadas por personas con mucho calor o excesivo frío, que derivan en mal de ojo, caída de mollera, vergüenza, etcétera. Luego, las de orden natural, que tienen alguna similitud o caen dentro de la clasificación internacional de enfermedades, aunque siempre subjetivadas desde la racionalidad del grupo, que a su vez pueden transitar desde las nociones de frío-caliente hasta las que sostiene la racionalidad biomédica, constituyéndose esta como el otro extremo del horizonte.

Lo que delimita entre lo natural y lo sobrenatural se localiza en la presencia de ciertos elementos particulares en el sueño.

Es que nosotros sabemos, o sea, cómo te voy a decir, ya sabemos qué puede sanar un Ch'abajel, a veces venimos: soñamos que tal cosa, que te pegaron, que te caíste. Entonces sabemos que hay que rezar, porque a nuestro ch'ulel lo molestan, pero eso llega a nuestro cuerpo, por eso sabemos bien cuando se necesita Ch'abajel (testimonio de Antón en Tenejapa).

Tal circunstancia orienta las estrategias hacia alguno de los polos, lo cual no significa que necesariamente se soslayen recursos de otros segmentos.

Sin embargo, por lo general, acorde con la subjetividad de los participantes, como en cualquier conjunto social, en principio se intenta resolver la enfermedad en el ámbito de la autoatención, predominantemente bajo el criterio frío-caliente. En este plano, como es el caso del mal de ojo, se recurre a manipulaciones corporales (masajes) o frotado con plantas; para otras condiciones, la ingestión de infusiones elaboradas con plantas medicinales locales o foráneas, o bien fármacos. Según esta lógica se recurre a la polaridad opuesta con el requerimiento de algún sabor también inscrito en alguna de las dos polaridades o neutro (amargo, agrio, dulce, salado y sin sabor), pero también con baños o pus 'baño de vapor'.

En la autoatención, bajo el mismo principio bipolar, las enfermedades son categorizadas en función de las manifestaciones físicas, emocionales o mentales (signos y síntomas); un ejemplo conciso sería que las enfermedades infecciosas del tracto respiratorio o digestivo con fiebre y escalofríos o sin estos, derivada de procesos infecciosos, inclinan hacia el polo frío las primeras y caliente las segundas.

Asimismo, las condiciones de orden socioeconómico como pobreza, solvencia económica, exclusión, violencia, marginación y racismo han adquirido peso en la 
racionalidad en que se sitúan los procesos mórbidos como factores que coadyuvan en la génesis y persistencia de las enfermedades naturales.

Es además en el plano de la autoatención donde se incorpora el «seudonaturismo», caracterizado por el uso de productos herbolarios procesados por corporaciones, principalmente internacionales, como Herbalife y Omnilife, entre otras.

Cuando el problema de salud rebasa las capacidades del plano de la autoatención se recurre a diferentes terapeutas, ya sea hierbateros, hueseros, promotores de salud, loktores o biomédicos a nivel público o privado.

Cuando «la enfermedad» pone en riesgo la vida desde un principio o ha persistido más allá de un tiempo en que las personas no sanan e incluso se agravan, las opciones, si es que de por medio se presentan sueños o simplemente por descartar, se busca la opinión y asistencia de etnomédicos; si no, como en el caso de la diabetes y otras enfermedades crónicas no transmisibles, la subjetividad, inducida por los mensajes biomédicos, tiende a inclinar las opiniones hacia el polo natural, atribuyendo, las personas que viven con diabetes, como causales directos e indirectos de la aflicción los excesos dietéticos (grasas y carbohidratos), el abuso en el consumo de refrescos y cerveza, o factores hereditarios, cuando se dice que el «azúcar» viene de la sangre.

Retomando el ámbito sobrenatural, cuando la enfermedad crónica o su agravamiento se asocia con sueños que la evocan, sea el enfermo el que sueña o algún familiar, o simplemente cuando otras opciones de atención a las que se ha recurrido no han ayudado a resolver el problema ya sea curando o controlando, dependiendo del caso, se llega a inferir que la causa se localiza en el plano sobrenatural, por lo que se recurre a los j-iloletik tsotsiles o ch'abajeletik tseltales, quienes tras pulsar para sentir/escuchar la sangre del enfermo determinarán si la enfermedad quiere rezo y ofrenda, o no, informando al enfermo y a su familia si se trata de enfermedad natural, o bien susto, pérdida del ch'ulel-alma, o ak'chamel, e indicarán en seguida el tipo de estrategia terapéutica que procede (véase esquema 1). 


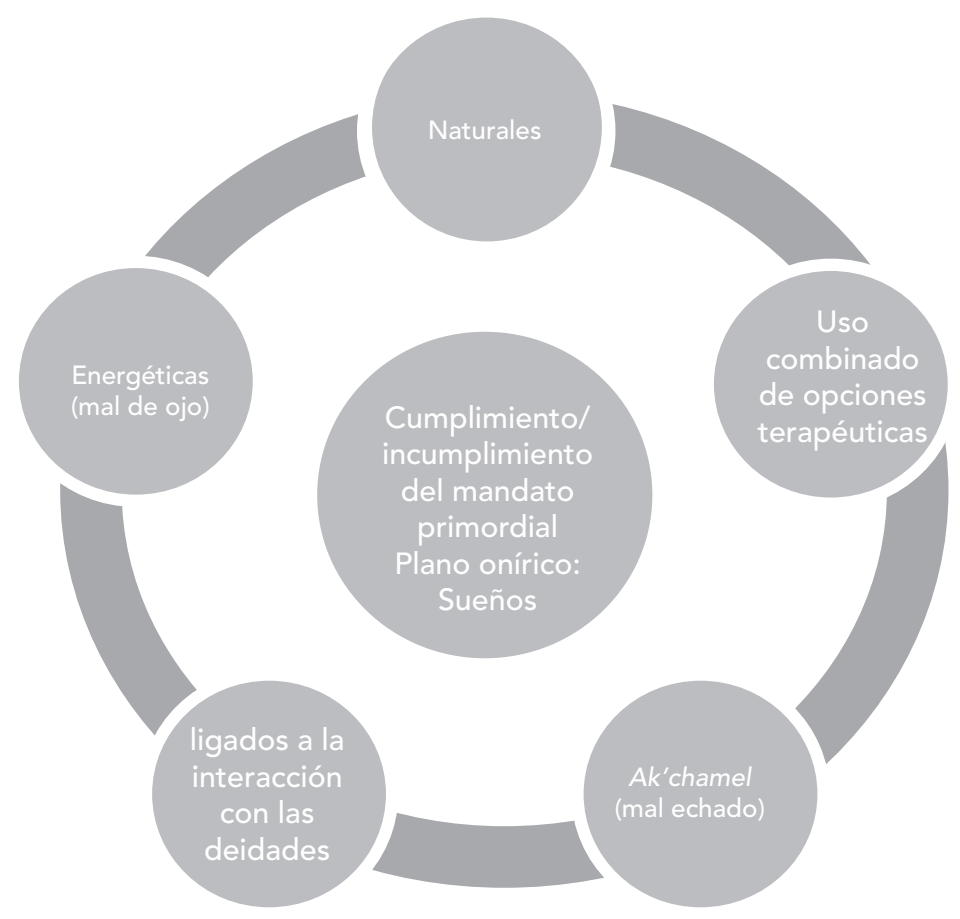

Fuente: Elaboración del autor.

La subjetividad referida al horizonte amplio se transfigura constantemente como resultado del impacto intersubjetivo en el que intervienen diferentes agentes de la sociedad global, entre los que predominan grupos religiosos con acción proselitista insidiosa, la publicidad farmacéutica vertida a través de los medios, el quehacer de los «merolicos» en calles y mercados, las estrategias callejeras de cooptación de clientes llevadas a cabo por entusiastas de las empresas de productos «herbolarios» y de «nutrición» como Omnilife y Herbalife y la insistente opinión de familiares y conocidos respecto de diferentes opciones y recursos.

En síntesis, en función de la pobreza o la solvencia económica del núcleo familiar, la conservación de la salud o el control de las enfermedades dependerá por un lado del cumplimiento de los aspectos de orden espiritual y religioso, nutrir y sostener a las deidades y, por otro, de llevar correctamente las prácticas de cuidado y respeto del cuerpo, la familia y la sociedad, evitando al máximo las confrontaciones y la envidia. 
La enfermedad surge cuando la armonía se ve alterada en el plano natural o espiritual, o en ambos, situándose cualquier padecimiento, según su gravedad o cronicidad, en cualquier parte del espectro, incluso alternadamente, con predominio del polo que la subjetividad del sujeto y su familia privilegien; por lo mismo, a esto se ligará indistintamente la atribución de la causa.

En tal sentido, la mayoría de las personas que viven con diabetes han transitado de ida y vuelta entre un ámbito y otro, o han combinado recursos terapéuticos de ambos polos, dependiendo de éxitos o fracasos atribuibles a las estrategias - las combinaciones de estas, de condiciones particulares de vida, así como de la influencia que en enfermos y familiares ejercen tanto los diferentes integrantes de sus redes como los agentes de los diferentes sistemas médicos y de empresas de productos «naturistas», al final inclinándose a largo plazo en alguno de los polos del horizonte.

Respecto de la diabetes, el balance se inclina claramente hacia el polo natural, ya que, por lo que documentamos en entrevistas y trayectorias, la mayor parte de los sujetos manifestaron, una vez que tuvieron «claridad» sobre su enfermedad, que el «azúcar no pide rezo, que es de origen natural y requiere en algunos casos plantas y en otros medicina de farmacia».

\section{Los casos}

Dado el reducido espacio de este artículo, se presentan solo dos casos respecto de los cuales los nombres y localidades han sido cambiados con objeto de mantener en el anonimato a quienes amablemente nos han abierto las puertas de sus hogares y su intimidad.

\section{Mira}

Este caso es el que más ha atraído nuestra atención. Mira tiene 51 años; es de apariencia igual a la cronológica, de constitución delgada y facies depresiva. No está inscrita en el seguro popular, por ser zapatista; es analfabeta y habla español con dificultad, pero más bien requiere traducción.

Radica en su propiedad donde vive desde que se casó por primera vez. Enviudó y está en una segunda relación desde hace 25 años; los hijos que tiene, un hom- 
bre y una mujer, provienen de su primer matrimonio. La casa en la que vive, que le fue heredada por sus padres, consta de siete habitaciones de bajareque con techo de lámina, tres de las cuales da en renta y una utiliza para vender frutas, verduras y textiles.

En lo que a religión toca, constantemente cambia, lo ha hecho como estrategia en la búsqueda de ayuda para los problemas de salud propios y de su hija; pero por su subjetividad y prácticas es más bien tradicionalista. Al momento de redacción de esta viñeta no acudía a ninguna iglesia.

Se dedica al comercio y además hace y vende tamales. Refirió que anteriormente su negocio estaba mejor surtido, pero que a partir de 2006, debido a gastos que realizó con motivo de la enfermedad crónica que sufre su hija, este se vino abajo.

De los dos hijos de Mira, la mujer, que vive con ella, al momento de la entrevista tenía 26 años de edad; el hijo, de quien no se detalla edad, salió de casa a los 12 años para irse a trabajar. Piensan que actualmente vive en Guadalajara y es militar. No se comunica con su madre, no envía ayuda monetaria ni de ningún tipo.

Mira enviudó aproximadamente en 1986, y por ahí del año 2000 apareció quien en la actualidad es su compañero, y que se fue metiendo a la casa poco a poco.

Mira calculó que inició con azúcar en 1997. Tenía mucha sed, por lo que tomaba mucha agua y orinaba a cada rato; además, estaba «muy débil». Para tratarse, primero tomó verbena y nopal, hasta que a los tres años se puso grave y buscó atención en la clínica de Esquipulas, en San Cristóbal de Las Casas, Chiapas. No sabe qué le dieron, solo que fueron inyecciones, porque también le estaba faltando la vista.

Considera que se enfermó del azúcar por tristeza y por efecto de violencia, debido, respecto de lo primero, al deceso de sus progenitores y su primer esposo; y lo segundo, a que a la muerte de sus padres un medio hermano por el lado paterno la sacó de la casa-terreno que tenía en su paraje de origen, de donde a su vez fue expulsada por la comunidad. Ahí fue cuando le empezó la enfermedad.

Entre los factores que a lo largo de los años han coadyuvado a una persistente hiperglucemia tenemos que en 2004, después de un parto, su hija enfermó gravemente de vómito, seguido de contracciones musculares tónico-clónicas generalizadas que no sanaron y que la incapacitaron, en esa etapa inicial, en 80\%; siguió siendo capaz de alimentarse, pero no de movilizarse sin ayuda, y preservó todas sus capacidades intelectuales y afectivas. Al parecer, la abuela materna murió de algo parecido. Dicho mal ha progresado y en noviembre de 2015 le 
provocó una incapacidad total no solo en torno a lo muscular sino también respecto de sus facultades mentales. La enfermedad no cuenta con un diagnóstico, a pesar de haber sido atendida en sus inicios por diferentes especialistas en el hospital de tercer nivel de Tapachula.

Aunado a lo anterior, Mira ha estado sometida durante años a golpizas por parte de su compañero actual, acción que realiza sin estar «tomado», además del estrés que genera la ambivalencia entre sacar al hombre de la casa y quedarse sin compañero a su edad, aun cuando este no aporta dinero ni en especie para el sostén de la familia, pero exige comida y sustento.

En el lapso no especificado y, según señala, debido al maltrato infligido por el marido y por «la maldad que ha cargado», Mira durante un año estuvo «echando trago» y en ese tiempo maltrataba a sus hijos aún pequeños. Lo pudo controlar debido a «que le pidió a diosito que la ayudara a dejarlo».

En algún momento de excesos del hombre solicitaron apoyo a las autoridades municipales para sacarlo de la casa, sin respuesta por parte de estas. Aducen que los funcionarios tienen miedo porque aquel hombre es zapatista.

Mira asegura que la persistencia de su azúcar y la enfermedad de su hija provienen de ak'chamel. Los poxtawanejetik 'los que ayudan a sanar', a los que ha recurrido reiteradamente, señalan al esposo como el responsable, que lleva a cabo el trabajo con objeto de que ambas mujeres, madre e hija, mueran y así quedarse con el terreno y la casa.

En repetidas ocasiones Mira ha sido amenazada por su esposo a punta de pistola, quien le advierte que si insiste en sacarlo de la casa la va a matar.

En el mes de mayo de 2011 el esposo se ausentó por varias semanas. Ella y su hija pensaron que finalmente se habían librado del hombre, pero nuevamente regresó amenazando con pistola a Mira y diciéndole que si no quería ser su esposa la iba a matar.

Mira ve en sueños que su marido está trabajando para dañarlas. También soñó a un rezador cancuquero (persona originaria de Cancuc, Chiapas), a quien fuera de sus sueños no conoce, que le dijo que su marido es el que le está haciendo daño a su hija.

A principios de 2011 la hija y el esposo fueron a vender a San Juan Cancuc. Mientras ella permanecía sentada en la camioneta, un hombre se acercó a preguntarle si podía caminar, y al responder ella que no, este le recomendó que visitara a un señor de Chiloljá, Cancuc, del que le dio santo y seña. En atención a 
los sueños sobre el cancuquero que su madre había tenido, visitaron a ese señor, quien les hizo 13 rezos y les confirmó que la maldad la puso el esposo de Mira.

Hacia agosto de 2011 buscaron a un rezador de Cañada Chica, Tenejapa, quien le dijo a Mira que su enfermedad no era diabetes real, sino ak'chamel, y le confirmó que quien les puso el mal fue el esposo y otra mujer que él tiene, y le aseguró que las sanaría. Sin embargo, a Mira le recomendó tomar medicamento para la diabetes hasta que se curara. También le prometió a su hija que la curaría. Sin embargo, después de algunas sesiones reconoció que a ella no le habían hecho ak'chamel, por lo que no iba a sanar.

Previo a la consulta con el Ch'abajel de Tenejapa, Mira, entre el 9 de junio de 2009 y septiembre de 2011, tuvo un promedio de glucosa plasmática casual de 323 mg/dl. El 21 de septiembre de 2011, al hacer la visita acostumbrada, me topé con que en su casa se estaba llevando a cabo una presentación de Herbalife. Mira estuvo muy animosa preparando los tres diferentes licuados que conforman la propuesta de esta empresa; el primero, según refirió el agente de Herbalife, consiste en aloe vera; el segundo, hierbas diversas y proteínas; y el tercero, vitaminas, minerales y fibra.

Previo a dicho evento, Mira asistió a un par de rezos con el ch'abajel tenejapaneco. Antes del evento de Herbalife, el día 14 del mismo mes, y aun asistiendo con el ch'abajel, registramos una lectura de glucosa plasmática casual de 173 $\mathrm{mg} / \mathrm{dl}$, casi la mitad del promedio arriba presentado. El día de la presentación de Herbalife, antes de la ingestión de los licuados de Herbalife, por primera vez desde que se inició el seguimiento, la glucemia en ayunas de Mira estuvo en 91 $\mathrm{mg} / \mathrm{dl}$.

Mira atribuyó dicho cambio, aunque aún no había probado los productos de Herbalife, a la ingesta de estos, soslayando totalmente el efecto del trabajo del ch'abajel. Motivada por ello se ausentó de la casa durante unos minutos para conseguir prestados los $\$ 700.00$ del costo del tratamiento completo.

Una vez terminado el contenido del primer tratamiento de Herbalife, Mira insistía en continuarlo, pero carecía de los recursos para la adquisición de los productos, dado su alto costo. Y como desde nuestro punto de vista los compuestos de dos de los licuados no contienen elementos conocidos que actúen sobre la diabetes, se le propuso a Mira que solo tomara el aloe, que se le consiguió a buen precio en San Cristóbal.

En el mes de marzo de 2012 nos enteramos, por boca de la hija, que en el paraje de donde el compañero de Mira es originario este fue acusado del asesinato 
de una persona, por lo que fue recluido en el Centro de Readaptación Social (CERESO) de San Cristóbal de Las Casas.

Durante el año que el hombre estuvo encarcelado, y solo con la ingestión de aloe vera, en las 10 siguientes glucometrías el promedio de la glucosa plasmática casual fue de $145 \mathrm{mg} / \mathrm{dl}$.

Al año, con la súbita aparición del hombre, quien fue liberado días antes y que reinició la violencia intrafamiliar incluso llegando a golpear en una ocasión a la hija discapacitada, la glucemia de Mira se elevó abruptamente. Eso derivó en su deseo de volver a consultar al ch'abajel, argumentando no haberlo hecho por falta de dinero. Hasta donde concluyó el seguimiento no había acudido con el ch'abajel y la hiperglucemia persistía.

\section{Much}

Much mujer tseltal de 63 años de edad que al menos aparentaba 10 años más; de complexión enjuta; monolingüe, analfabeta, no asistió a la escuela porque su padre pensaba "que los profesores se la iban a comer»; de usos y costumbres tradicionales. Murió a principios de 2015 por complicaciones de la diabetes.

En el predio de aproximadamente 1000 metros en que residía, tienen sus viviendas los suegros (quienes aún viven) y todos sus hijos. Ella compartía su casa con una nieta que es sorda de nacimiento. Durante los últimos años de vida contó con un ingreso de $\$ 790.00$ bimensual proveniente del programa Oportunidades, con el que sobrevivían ambas; con eso y los productos de su milpa, que en ocasiones daba para surtir de maíz todo el año, completaban su alimentación.

La poca ayuda que recibía de sus hijos no incluía apoyo económico, ni lo relacionado con gasto para la búsqueda de atención o medicación para el control de la diabetes.

Much vinculaba su enfermedad a una vida de extremo sufrimiento. Entre los aspectos que destacan de su narrativa resalta que su padre fue asesinado cuando ella tenía la edad de ocho años. Además de la tristeza que esto le generó, dicho evento situó a la familia en pobreza extrema e intenso trabajo.

Al decir de Much su padecimiento inició en la juventud; estando recién casada empezó con un fuerte dolor abdominal (sme 'jwinik) que fue atribuido a ak'chamel, echado por una mujer que quedó muy enojada y envidiosa porque Much se quedó con el hombre que ella también deseaba. 
Supo que era ak'chamel porque en tres ocasiones arrojó huevecillos de rana. Para sanar acudió con diferentes jpoxtawanej, incluso llegó hasta Pujiltic, en Carranza, en busca de atención. Todas las personas a las que consultó le dijeron que esa mujer le había puesto el mal.

En una ocasión, estando su esposo ausente, aprovechó para hacerle una visita.

Le fui a decir: «¿Qué fue lo que te hice?» Le dije que la iba a ir a matar. «No me importa si voy a la cárcel con todo y hueso, aunque la verdad no sé si me puedan encarcelar todavía, así como estoy». Fue lo que dije.

Al poco tiempo se le quitó el dolor, pero el mal ahí quedó.

Contó que desde joven su vida estuvo plagada de disgustos, que junto con su esposo se embriaga con frecuencia y en dicho estado reñían en demasía. Destacó el hecho de que sus suegros «eran malos», siempre la regañaban, no le daban de comer suficiente, motivo por el cual reñía con su esposo, reclamando que ella estaba acostumbrada a comer bien y no a pasar hambres — lo que ciertamente se contradice con su decir de pobreza y sufrimiento-.

Un hecho relevante que tocó su vida de manera profunda fue que en una ocasión recibió una bala de uno de sus hijos. El suceso ocurrió después de un rezo, estando presentes quien fuera su esposo, ella, el hijo, la nieta y la nuera. La esposa del hijo discutía acaloradamente con su marido una situación de celos con la nieta sordomuda. En una de esas este tomó un rifle que estaba a mano, al lado del altar, y disparó con la intención de matar a la nieta. El balazo penetró en el vientre de Much. Otro de sus hijos y su esposa, quienes no participaban en el rezo, llevaron a doña Much al hospital; la bala no dañó vísceras ni órganos, solo músculo. Le sacaron la bala a un lado de la espina baja.

Según señaló Much, a su esposo «no se le salió el cólico», considerando que eventualmente eso fue lo que 15 días después lo llevó a la muerte. Sin embargo, lo que en realidad sucedió, según relató uno de los hijos, fue que el esposo se puso mal durante la fiesta de San Diego y al irse a casa se desorientó totalmente. Lo llevaron al centro de salud, donde le detectaron que tenía la presión arterial muy elevada. Pero no aceptó el tratamiento que le ofrecían y acudió con un señor, quien en un rato le aplicó dos litros de suero vitaminado, «con eso se mareó mucho y al rato se murió».

Meses después el hijo que le produjo la herida a Much regresó al predio donde tiene su casa. De hecho, la mejor casa es la de él. 
Por otro lado, Much vivía constantemente abrumada por problemas derivados de pleitos y abusos entre sus hijos. Poco antes de fallecer se enteró de que uno de ellos estaba vendiendo un terreno que le pertenecía a ella, debido a lo cual se armó un lío entre los hermanos. En otra ocasión los hijos se liaron a golpes estando sobrios. Ellos le decían que no pusiera atención, ya que los conflictos rápidamente se resolvían, y que mejor se fuera a la milpa, que era donde se tranquilizaba.

Siete años después de que se curó del dolor arriba referido, es decir, ocho años antes de la entrevista, este nuevamente se presentó, pero acompañado de boca seca, sed excesiva, poliuria, debilidad y visión borrosa. De nueva cuenta atribuyó la causa de su padecimiento a mal echado, aferrándose a tal criterio bajo el razonamiento de que no había medicamento que se lo quitara, ni siquiera que lo controlara. Refirió que visitó infinidad de médicos y que ninguno logró aliviarla.

Nunca pudo constatar quién le puso el «daño», si la misma mujer u otra persona, porque debido a la falta de dinero no pudo preguntar a quienes le podían resolver la incógnita.

Se enteró de que padecía de "azúcar» cuando en una ocasión que se puso grave la llevaron a la Clínica de Esquipulas en San Cristóbal de Las Casas, donde le detectaron una glucemia de 540 mg/dl, y la canalizaron al Hospital de las Culturas, donde permaneció internada durante tres días. No supo qué medicamentos le suministraron, solo recordaba que lo hicieron por vía intravenosa. A partir de ahí le instituyeron tratamiento a base de pastillas, según pude ver, metformina de 850 mg y glibenclamida de 5 mg, más vitaminas, todas las cuales tomaba dos veces al día, por la mañana y a mediodía, lo que le producía dolor de estómago: «no se quita y arde como chile». Recibía sus medicamentos en la UMR. Respecto de esta se quejaba de que la atención de los médicos que venían por Oportunidades era pésima y además cuando les pedía medicamento no se lo daban. Complementaba su tratamiento con hierba mora, y otras de sabor amargo. También intentó controlarse con aloe vera, pero sin éxito.

Respecto de sus hijos, refirió que ninguno se preocupaba por ella, que no les importaba que estuviera enferma, y que dada su condición de anciana monolingüe no se aventuraba a ir sola a la clínica. Se quejaba de que al estar en casa sufría de dolores e incomodidad. Todo eso se le quitaba cuando se salía a caminar o a trabajar en la milpa.

Dormía unas cuantas horas por la noche y luego ya no conciliaba el sueño. 
El promedio de glucosa plasmática casual resultado de 10 tomas entre agosto de 2011 y enero de 2012 fue de 402 mg/dl. Una de las lecturas arrojó 146 mg/ dl, lo que tuvo lugar un día después de que Much se embriagara con pox, una práctica que no abandona aunque no lo hace con mucha frecuencia.

Durante los meses que visité a Much solicité sin ningún éxito a sus hijos que le brindaran el apoyo que requería para controlarse. Sin embargo, me pedían que no dejara de verla, aunque mi visita en poco o en nada la beneficiara.

\section{Consideraciones finales}

Del total de casos trabajados en las tres cabeceras municipales, seis sujetos consideraron que la causa directa del «azúcar» que padecían fue ak'chamel, en tanto que solo una lo consideró como desencadenante. En todos se trató de mujeres mayores de 45 años, monolingües y analfabetas, y prevalecía la asociación con el mal echado basada en ciertos elementos presentes en sus sueños, aunada a condiciones particulares de vida caracterizada por violencia constante. Tal apreciación, a su vez, en dichos casos fue corroborada por los j-iloletik o ch'abajeletik, además de indicar que el móvil del ak'chamel es principalmente la envidia.

Cabe destacar que la mayoría de los sujetos participantes situaron la diabetes mellitus en el ámbito natural, «que no lleva rezo». Todos provienen de círculos familiares situados preferentemente en cabeceras municipales, lo cual significa mayor interacción con la sociedad regional y global, menores índices educativos, sobre todo entre los jóvenes, todo ello en comparación con sus pares que viven en «la montaña», en cuyas dinámicas se considera importante la atención y el cuidado de los enfermos, y por tanto su intersubjetividad repercute en las nociones y la elección de las terapéuticas, con una inclinación predominante, como ya se indicó, hacia los recursos biomédicos y herbolarios.

Dentro de este espectro, considerando, además, que la diabetes mellitus es una enfermedad que suele presentarse después de los 50 años, la mayoría de mujeres tsotsiles y tseltales asentadas en sus municipios de origen, a partir de este estamento, son predominantemente monolingües, analfabetas, y tradicionalistas, con mayor inclinación en dicho sentido entre mayor lejanía y menores comunicaciones haya entre el centro y sus localidades. En ese tenor, frecuentemente nos topamos con parejas o mujeres viudas que reciben poca o ninguna atención por parte de sus familiares, aun cuando llegan a compartir el mismo 
predio, como en el caso de Much, lo que determina una racionalidad que no va más allá de la visión tradicionalista que implica una inclinación en la que suelen adjudicarse las causas de la diabetes a envidia y ak'chamel.

Si bien el número de casos que registramos con esta orientación no es significativo, no hay que soslayar que el universo de trabajo se centró en las cabeceras municipales, de ahí que esta temática no resulte tangencial, sino que constituye un llamado a poner atención en dicha situación y eventualmente buscar soluciones.

Respecto del horizonte amplio, cabe mencionar que es en la esfera de la transubjetividad donde se sitúan las racionalidades, los discursos y las acciones provenientes de la tradición, la religión, la globalidad, pero, sobre todo, las de los diferentes sistemas de atención a la salud-enfermedad.

En el plano de la intersubjetividad tiene lugar el intercambio de la experiencia acumulada por los conjuntos sociales respecto de la laxitud en el uso de diferentes recursos, con propuestas de distintas opciones de manejo, limitaciones y beneficios de los recursos.

Así, al subjetivar, los sujetos actúan sin restricciones en el uso de las diversas opciones, centrando la búsqueda en el plano de la eficacia, sin cuestionar racionalidades.

Cabe destacar, por otro lado, que en los perfiles de vida de las mujeres a que hacemos referencia están presentes constantes alteraciones emocionales resultado de la violencia recurrente de distintos órdenes que padecen a lo largo de sus vidas, entre las que destacan tristeza, enojo, pero lo más grave, resignada contención de dichas emociones, aspectos que resaltaron de manera explícita en varias visitas.

El otro elemento de carácter central que conlleva sufrimiento emocional, sobre todo para la mujer, es el referido a la violencia social y estructural enmarcada en contextos de pobreza, de los que destaca entre todas por su carácter, podríamos decir epidémico, la violencia intrafamiliar: golpizas, regaños, gritos, maltrato, sobrecarga en la labores domésticas y de producción, principalmente entre los grupos mayas de los Altos, con todas las implicaciones que esto conlleva, además de inequidad: marginación social, discriminación, sobre-explotación, inestabilidad en el trabajo, percepciones económicas raquíticas, desempleo, mala calidad de la atención médica, servicios sanitarios insuficientes.

Por tanto, las emociones adquieren relevancia en el desencadenamiento, agravamiento y desenlace de la diabetes mellitus. En lo concerniente a ese aspecto, 
una y otra vez reportado en las narrativas, se considera que si bien las emociones no son la causa directa de la diabetes, se constituyen como causa desencadenante y como una constante agravante. En este sentido, en los casos presentados y en lo documentado a lo largo de la investigación nos hemos invariablemente topado con un evento clave de sufrimiento o de violencia social que el narrador atribuye como causa y perpetuación de la diabetes.

Otro elemento de sufrimiento remite a la aprensión que genera la necesidad de romper el control dietético, en aras de satisfacer las apetencias personales, o bien cumplir con la exigencia comensal que los cargos religiosos o políticos demandan.

Adjudicar la causalidad al ak'chamel implica la consideración de que una vez erradicado el mal echado, la enfermedad se curará, es como asumir que la aflicción no pertenece al cuerpo, sino que es algo puesto, ajeno, que debe eliminarse. Lo cual, se sabe, no es sencillo, toda vez que necesariamente se requiere la intermediación de alguien que cuente con recursos suficientes para dominar y erradicar. Y a mayor poder, más elevado será el costo de la operación, lo cual, como se ha visto, queda fuera de las posibilidades de Mira y Much.

En relación con lo anterior, en el sentido de considerar que la enfermedad responde a un «objeto» puesto, que puede ser medianamente controlado con fármacos, plantas y rezos sin fuerza erradicadora, derivó en que ninguna de las personas que se pensaron afectadas por ak'chamel dio validez o consideró que por el hecho de consumir los alimentos que se proscriben en las recomendaciones dietéticas, sobre todo los calificados como chatarra y los refrescos, contribuyan al desencadenamiento y/o agravamiento de la DM, así como a la aparición de complicaciones, por lo que sobre todo eran dadas a la frecuente ingestión

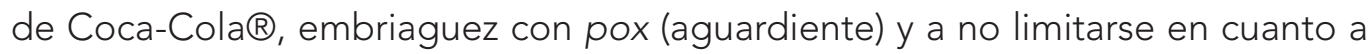
alimentos ricos en carbohidratos y grasas.

Para concluir, faltaría señalar la facilidad con que se pasa de una opción terapéutica a otra, o su uso simultáneo, y precisar que la presencia de sueños o la pulsación no se constituyen como barreras entre lo natural y lo sobrenatural, lo que destaca, por ejemplo, cuando un ch'abajel, en cuanto logra la esperada erradicación del mal puesto y en consecuencia la curación, instruye a su paciente a continuar con el tratamiento, ya sea biomédico o herbolario. Si bien nadie explica cómo se constituyen los criterios para ello, en la práctica nos indica la gran flexibilidad, ante un panorama inhóspito para la vida, para la solución de las enfermedades y de cercanía con la muerte, de recurrir sin obstáculos a todo 
aquello que ha probado tener efectos benéficos en la mejora o control de la diabetes mellitus.

\section{Fuentes de consulta}

Bartolomé, Miguel, Alicia Barabas, 1996, La pluralidad en peligro, México, Instituto Nacional de Antropología e Histyoria-Instituto Nacional Indigenista (Regiones de México).

Biehl, J., Byron Good, Arthur Kleinman, 2007, «Introduction, rethinking subjectivity», en Biehl, J., Byron Good, Arthur Kleinman (coords.), Subjectivity: ethnographic investigations, Berkeley, University of California Press.

Fabrega, Horacio Jr., Daniel B. Silver, 1973, Illness and shamanistic curing in Zinacantan, EUA, Stanford University Press.

Figuerola, Helios, 2010, Los dioses, los hombres y las palabras. En la comunidad de San Juan Evangelista, Cancuc en Chiapas, México, Centro Peninsular en Humanidades y Ciencias Sociales de la Universidad Nacional Autónoma de México.

German, Michael, 2003, «Regulación del gen de la insulina», en Le Roith, Derek, Simeon Taylor, Jerrold M. Olefsky (coords.), Diabetes mellitus. Texto básico y clínico, México, McGraw Hill, pp. 13-23.

Good-Eshelman, C., 2015, «Las cosmovisiones, la historia y la tradición intelectual en Mesoamérica», en Alejandra Gámez y Alfredo López-Austin (coords.), Cosmovisión mesoamericana. Reflexiones, polémicas y etnografías, México, El Colegio de México/ Fondo de Cultura Económica/Benemérita Universidad Autónoma de Puebla.

Gossen, Gary, 1980, Los chamulas en el mundo del Sol. Tiempo y espacio en una tradición oral Maya, México, Consejo Nacional para la Cultura y las Artes/Instituto Nacional Indigenista.

_- 1975, «Animal souls and human destiny in Chamula», Man 10. pp. 448-461.

Guiteras Holmes, Calixta, 1965, Los peligros del alma, México, Fondo de Cultura Económica.

Hermitte M., Esther, 1970, Poder sobrenatural y control social en un pueblo maya Contemporáneo, Chiapas, Consejo Estatal de Fomento a la Investigación y la Difusión de la Cultura (Cuadernos Municipales).

Holland R., William, 1963, Medicina maya en los altos de Chiapas: Un estudio del cambio socio-cultural, México, Instituto Nacional Indigenista.

Instituto de Salud de Chiapas (ISCH), 2008, Mortalidad General Chiapas 2008, Secretaría de Salud/Gobierno del Estado de Chiapas, disponible en <www.salud.chiapas.gob. mx> [consulta: 24/05/2010].

Instituto Nacional de Estadística y Geografía (INEGI), 2006, Anuario Estadístico y Geográfico de Chiapas, México, INEGI-Gobierno del Estado de Chiapas.

- 2009, Anuario Estadístico y Geográfico de Chiapas, México, INEGI-Gobierno del Estado de Chiapas. 
International Diabetes Federation (IDF), 2009, Who we are, disponible en <http://www.idf. org/webdata/docs/IDF-leaflet-2009.pdf> [consulta: 18/05/2010].

—_, 2012, «Diabetes Federation Poster 2012_es», IDF Atlas, disponible en <http://www. idf.org/sites/default/files/5E_IDFAtlasPoster_2012_ES.pdf> [consulta: 08/10/2013].

_ 2013, Atlas de la diabetes de la IDF, International Diabetes Federation (IDF), disponible en <http://www.idf.org/diabetesatlas/sp_6E_Atlas_Full.pdf>.

— 2015 Atlas de la diabetes de la IDF, International Diabetes Federation (IDF), disponible en <http://www.fundaciondiabetes.org/upload/publicaciones_ficheros/95/ mensajes_clave.jpg $>$.

Jodelet, Denise, 2008, «El movimiento de retorno al sujeto y el enfoque de las representaciones sociales», Cultura y representaciones sociales. Un espacio para el diálogo transdisciplinario, Instituto de Investigaciones Sociales-UNAM, 3(5) septiembre, disponible en <www.ojs.unam.mx/index.php/crs/article/download/16356/1556>.

Köhler, Ürlich, 1995, Chonbilal ch'ulelal, alma vendida. Elementos fundamentales de la cosmología y religión mesoamericanas en una canto maya-tzotzil, México, Instituto de Investigaciones Antropológicas, Universidad Nacional Autónoma de México.

Menéndez, Eduardo, 2003, «Modelos de atención de los padecimientos: de exclusiones teóricas y articulaciones prácticas», Ciencia \& Saude Colectiva, 8 (1): pp. 185-207.

Page Pliego, Jaime, 2010, Yombil «Puesta su flor en el altar». Una mirada a la etnomedicina en Oxchuc, Chiapas a partir de los j-poxtawanejetik organizados, México, Programa de Investigaciones Multidisciplinarias sobre Mesoamérica y el SuresteUniversidad Nacional Autónoma de México.

_- 2011 (reimpresión digital 2005), El mandato de los dioses: Medicina entre los tzotziles de Chamula y Chenalhó, Chiapas, México, Programa de Investigaciones Multidisciplinarias sobre Mesoamérica y el Sureste-UNAM (Serie Científica, 11).

Pitarch, Pedro, 1996, Ch'ulel: una etnografía de las almas tzeltales, México, Fondo de Cultura Económica.

Popol Vuh, 1961 (1952), Antiguas historias del Quiché, Adrián Recinos (trad.), México, Fondo de Cultura Económica.

Pozas Arcineaga, Ricardo, 1977, Chamula, México, Instituto Nacional Indigenista (Clásicos de la Antropología Mexicana, 1-II). 